\title{
Pacific
}

Journal of

Mathematics

\section{GLOBAL CLASSICAL SOLUTIONS TO HYPERBOLIC GEOMETRIC FLOW ON RIEMANN SURFACES}

FAGUi LiU AND YUANZHANG ZHANG 


\title{
GLOBAL CLASSICAL SOLUTIONS TO HYPERBOLIC GEOMETRIC FLOW ON RIEMANN SURFACES
}

\author{
FAGUI LIU AND YUANZHANG ZHANG
}

\begin{abstract}
We consider the Cauchy problem for hyperbolic geometric flow equations introduced recently by Kong and Liu motivated by the Einstein equation and Hamilton Ricci flow, and obtain a necessary and sufficient condition for the global existence of classical solutions to this kind of flow on Riemann surfaces. The results show that the scalar curvature of the solution metric $g_{i j}$ converges to one of flat curvature, and the hyperbolic geometric flow has the advantage that the surgery technique may be replaced by choosing a suitable initial velocity tensor.
\end{abstract}

\section{Introduction}

Let $M$ be an $n$-dimensional complete Riemann manifold with Riemann metric $g_{i j}$. The general evolution equation

$$
\frac{\partial^{2} g_{i j}}{\partial t^{2}}+2 R_{i j}+\mathscr{F}\left(g, \frac{\partial g}{\partial t}\right)=0
$$

for the metric $g_{i j}$ has been recently introduced by Kong and Liu [2007] and called the generalized hyperbolic geometric flow (denoted by HGF). Here $\mathscr{F}$ are some given smooth functions of the Riemann metric $g$ and its first derivative with respect to $t$, and $R_{i j}$ are the components of the Ricci curvature tensor. In this paper, we may the HGF the Kong-Liu hyperbolic geometric flow. The local existence and nonlinear stability have been proved by Dai, Kong and Liu [ $\geq 2010 ; 2008]$. See the celebrated survey paper [Kong 2008] for progress on this new topic.

In this paper, we study the evolution of a Riemann metric $g_{i j}$ on a Riemann surface $M$ by its Ricci curvature tensor $R_{i j}$ under the HGF equation

$$
\frac{\partial^{2} g_{i j}}{\partial t^{2}}=-2 R_{i j}
$$

MSC2000: primary 30F45, 58J45; secondary 35L65, 35L45.

Keywords: hyperbolic geometric flow, Riemann surface, quasilinear hyperbolic system, classical solution.

Supported by the NSF of China (grant 10571024) and the Basic Research of Henan Province. 
Here, we are interested in the initial metric

$$
d s^{2}=u_{0}(x)\left(d x^{2}+d y^{2}\right) \quad \text { at } t=0
$$

on a surface of topological type $\mathbb{R}^{2}$, where $u_{0}(x)$ is a $C^{2}$ function with bounded $C^{2}$ norm and satisfies

$$
0<k \leq u_{0}(x) \leq M<\infty,
$$

where $k$ and $M$ are positive constants.

On this surface, the HGF equation simplifies because all of the information about curvature is contained in the scalar curvature function $R$. In our notation, $R=2 K$, where $K$ is the Gauss curvature. The Ricci curvature is given by $R_{i j}=\frac{1}{2} R g_{i j}$, so the HGF equation simplifies to

$$
\frac{\partial^{2} g_{i j}}{\partial t^{2}}=-R g_{i j}
$$

The metric for a surface can always be written (at least locally) as $g_{i j}=u(t, x, y) \delta_{i j}$, where $u(t, x, y)>0$, and $\delta_{i j}$ is Kronecker's symbol. Therefore, we have

$$
R=-\frac{\Delta \ln u}{u} .
$$

Thus, Equation (3) reduces to $u_{t t}-\Delta \ln u=0$.

The initial data $u_{0}(x)$ depends only on $x$ and not $y$; therefore, we may consider the Cauchy problem

$$
\left\{\begin{array}{c}
u_{t t}-(\ln u)_{x x}=0, \\
u=u_{0}(x) \quad \text { at } t=0, \\
u_{t}=u_{1}(x) \quad \text { at } t=0,
\end{array}\right.
$$

where $u_{1}(x) \in C^{1}$ with bounded $C^{1}$ norm.

By using the transformation

$$
\phi=\ln u,
$$

Kong and Liu have proved the following theorem.

Theorem 1.1 [Kong et al. 2009]. Suppose that $u_{1}(x) \geq\left|u_{0}^{\prime}(x)\right| / \sqrt{u_{0}(x)}$ for all $x \in \mathbb{R}$. Then, the Cauchy problem (5) admits a unique global solution for all $t \in \mathbb{R}$.

Moreover, if $u_{1}(x) \equiv u_{0}^{\prime}(x) / \sqrt{u_{0}(x)}$, and there exists a point $x_{0} \in \mathbb{R}$ such that $u_{0}^{\prime}\left(x_{0}\right)<0$, then the Cauchy problem (5) admits a unique classical solution only in $[0, T) \times \mathbb{R}$, where

$$
T=-\frac{2}{\inf _{x}\left\{u_{0}^{\prime}(x) u_{0}^{-3 / 2}(x)\right\}} .
$$

In this paper, we will prove the following theorems without using (6). 
Theorem 1.2. Suppose that

$$
\begin{aligned}
& u_{1}(x)+\frac{u_{0}^{\prime}(x)}{\sqrt{u_{0}(x)}} \geq 0 \quad \text { for all } x \in \mathbb{R}, \\
& u_{1}(x)-\frac{u_{0}^{\prime}(x)}{\sqrt{u_{0}(x)}} \geq 0 \quad \text { for all } x \in \mathbb{R} .
\end{aligned}
$$

Then (5) admits a unique global solution for all $t \in \mathbb{R}$.

Theorem 1.3. If there exists a point $x_{0} \in \mathbb{R}$, such that

$$
u_{1}\left(x_{0}\right)+\frac{u_{0}^{\prime}\left(x_{0}\right)}{\sqrt{u_{0}\left(x_{0}\right)}}<0,
$$

or there exists a point $x_{0} \in \mathbb{R}$ such that

$$
u_{1}\left(x_{0}\right)-\frac{u_{0}^{\prime}\left(x_{0}\right)}{\sqrt{u_{0}\left(x_{0}\right)}}<0
$$

then (5) admits a unique classical solution only in $[0, T) \times \mathbb{R}$.

Remark 1.4. Following Theorem 1.2, it is easy to see that Cauchy problem

$$
\begin{cases}\frac{\partial^{2} g_{i j}}{\partial t^{2}}=-2 R_{i j} & \text { for } i, j=1,2, \\ g_{i j}=u_{0}(x) \delta_{i j} & \text { for } t=0, \\ \frac{\partial g_{i j}}{\partial t}=u_{1}(x) \delta_{i j} & \text { for } t=0 \text { and } i, j=1,2\end{cases}
$$

has a unique smooth solution for all time $t \in \mathbb{R}$, and the solution metric $g_{i j}$ assumes the form

$$
g_{i j}=u(x, t) \delta_{i j} \quad \text { for } i, j=1,2 .
$$

Theorems 1.2 and 1.3 will be proved in Sections 3 and 4. Using Theorem 1.2, we can further prove this:

Theorem 1.5. Suppose that

$$
\inf _{x}\left\{u_{1}(x)+\frac{u_{0}^{\prime}(x)}{\sqrt{u_{0}(x)}}\right\}>0 \quad \text { and } \quad \inf _{x}\left\{u_{1}(x)-\frac{u_{0}^{\prime}(x)}{\sqrt{u_{0}(x)}}\right\}>0 .
$$

Then (11) has a unique classical solution of the form (12) for all time. Moreover the scalar curvature $R(x, t)$ corresponding to the solution metric $g_{i j}$ satisfies

$$
R(x, t) \rightarrow 0 \text { as } t \rightarrow+\infty,
$$

and there exists positive constant $k_{1}>0$ independent of $t$ and $x$ such that

$$
R(x, t) \leq k_{1} \quad \text { for all }(t, x) \in \mathbb{R}^{+} \times \mathbb{R} .
$$


Theorem 1.5 shows that any metric on a simply connected noncompact surface converges to one of flat curvature by choosing a suitable initial velocity tensor $\partial g_{i j}(x, 0) / \partial t$, but the general case of a metric in $\mathbb{R}^{2}$ is still open. Theorem 1.3 shows that if we do not choose suitable a initial velocity tensor $\partial g_{i j}(x, 0) / \partial t$, the solution to Cauchy problem will blow up in finite time.

\section{Preliminaries}

We need only discuss the classical solution on $t \geq 0$. The result for $t \leq 0$ can be obtained by changing $t$ to $-t$.

Let

$$
u_{t}=v \quad \text { and } \quad u_{x}=w .
$$

Then, it follows from the first equation in (5) and (14) that

$$
u_{t}=v, \quad w_{t}-v_{x}=0, \quad v_{t}-(1 / u) w_{x}=-w^{2} / u^{2} .
$$

It is easy to see that the eigenvalues of equations (15) are

$$
\lambda_{1}=-\lambda, \quad \lambda_{2}=0, \quad \lambda_{3}=\lambda, \quad \lambda=1 / \sqrt{u},
$$

and the matrix $L(U)$ (where $U=(u, w, v)^{T}$ ) of left eigenvectors and the matrix $R(U)$ of right eigenvectors are respectively

$$
\begin{aligned}
& L(U)=\left(\begin{array}{l}
l_{1}(U) \\
l_{2}(U) \\
l_{3}(U)
\end{array}\right)=\left(\begin{array}{ccc}
0 & \lambda & 1 \\
1 & 0 & 0 \\
0 & -\lambda & 1
\end{array}\right), \\
& R(U)=\left(r_{1}(U), r_{2}(U), r_{3}(U)\right)=\left(\begin{array}{ccc}
0 & 1 & 0 \\
\lambda & 0 & -\lambda \\
1 & 0 & 1
\end{array}\right) .
\end{aligned}
$$

Since $\nabla \lambda_{i}(U) r_{i}(U) \equiv 0$ for $i=1,2,3,(15)$ is a linear degenerate strict hyperbolic system.

Set

$$
p=v+\lambda w \quad \text { and } \quad q=v-\lambda w .
$$

Lemma 2.1. $p$ and $q$ satisfy

$$
\begin{aligned}
p_{t}-\lambda p_{x} & =\frac{1}{4} \lambda^{2} p(q-p), \\
u_{t} & =\frac{1}{2}(p+q), \\
q_{t}+\lambda q_{x} & =\frac{1}{4} \lambda^{2} q(p-q) .
\end{aligned}
$$


Proof. Noting that $\lambda_{t}=-\frac{1}{2} \lambda^{3} v$ and $\lambda_{x}=-\frac{1}{2} \lambda^{3} w$, we calculate

$$
\begin{aligned}
p_{t}-\lambda p_{x} & =(v+\lambda w)_{t}-\lambda(v+\lambda w)_{x} \\
& =v_{t}-\lambda v_{x}+\lambda\left(w_{t}-\lambda w_{x}\right)+w\left(\lambda_{t}-\lambda \lambda_{x}\right) \\
& =-\frac{1}{2} \lambda^{3} w(v+\lambda w)=\frac{1}{4} \lambda^{2}(q-p) p .
\end{aligned}
$$

In a similar way, we can prove (17c), and (17b) is obvious.

Let

$$
r=p_{x}+\frac{1}{8} \lambda p q \quad \text { and } \quad s=q_{x}-\frac{1}{8} \lambda p q .
$$

Lemma 2.2. $r$ and $s$ satisfy

$$
\begin{aligned}
& r_{t}-\lambda r_{x}=\frac{1}{4} \lambda^{2}(2 q-3 p) r+\frac{1}{32} \lambda^{3} p^{2}(4 p-5 q), \\
& s_{t}+\lambda s_{x}=\frac{1}{4} \lambda^{2}(2 p-3 q) s+\frac{1}{32} \lambda^{3} q^{2}(5 p-4 q) .
\end{aligned}
$$

Proof. Let

$$
L_{1}=\frac{\partial}{\partial t}-\lambda \frac{\partial}{\partial x} \quad \text { and } \quad L_{2}=\frac{\partial}{\partial t}+\lambda \frac{\partial}{\partial x} .
$$

Then,

$$
\begin{aligned}
& L_{1} p_{x}=\frac{1}{4} \lambda^{2}\left((2 q-3 p) p_{x}+p q_{x}\right)+\frac{1}{8} \lambda^{3} p(p-q)^{2}, \\
& L_{2} q_{x}=\frac{1}{4} \lambda^{2}\left((2 p-3 q) q_{x}+q p_{x}\right)-\frac{1}{8} \lambda^{3} q(p-q)^{2} .
\end{aligned}
$$

Noting that

$$
\begin{aligned}
L_{1}(\lambda p) & =-\frac{1}{4} \lambda^{3} p(q+p), & L_{2}(\lambda q) & =-\frac{1}{4} \lambda^{3} q(p+q), \\
L_{1} q & =-\frac{1}{16} \lambda^{3} p q^{2}-\frac{1}{8} L_{1}(\lambda p q), & L_{2} p & =\frac{1}{16} \lambda^{3} q p^{2}+\frac{1}{8} L_{2}(\lambda p q),
\end{aligned}
$$

by a direct calculation, we easily prove (18) and (19).

Noting that (5), (14) and (16), we write at $t=0$

$$
\begin{aligned}
& p=p_{0}(x) \equiv u_{1}(x)+\lambda_{0}(x) u_{0}^{\prime}(x), \quad u=u_{0}(x), \\
& q=q_{0}(x)=u_{1}(x)-\lambda_{0}(x) u_{0}^{\prime}(x),
\end{aligned}
$$

where $\lambda_{0}(x)=1 / \sqrt{u_{0}(x)}$.

Theorem 2.3 (the existence domain $D(T)$ of the classical solution to (5)). If there exists a positive constant $M_{1}$ such that

$$
0 \leq p(x, t) \leq M_{1} \quad \text { and } \quad 0 \leq q(x, t) \leq M_{1},
$$

then on $D(T)$,

$$
\begin{aligned}
|u(x, t)| & \leq M(T), & \left|u_{x}(x, t)\right| & \leq M(T), \quad\left|u_{t}(x, t)\right| \leq M(T), \\
|r(x, t)| & \leq M(T), & |s(x, t)| & \leq M(T),
\end{aligned}
$$


where $M(T)$ is a positive constant, and

$$
D(T)=\{(x, t) \mid x \in \mathbb{R}, 0 \leq t \leq T, T>0\} .
$$

Then, by the local existence theorem of the classical solution to quasilinear hyperbolic systems, (5) admits a unique global classical solution on $t \geq 0$.

Remark 2.4. Theorem 2.3 shows that if a singularity occurs in a $C^{1}$ solution to Cauchy problem (17) and (20) in finite time, then the solution itself should go to infinity at the starting point of the singularity [Li and Liu 2003].

Proof of Theorem 2.3. Through any point $(t, x)$, let

$$
x=x_{1}\left(t, \beta_{1}\right), \quad x=x_{2}\left(t, \beta_{2}\right), \quad x=x_{3}\left(t, \beta_{3}\right)
$$

be the $\lambda_{1}, \lambda_{2}$ and $\lambda_{3}$ characteristics, respectively, that satisfy

$$
\begin{aligned}
& \frac{d x_{1}}{d t}=\lambda_{1}=-\lambda, \quad \frac{d x_{2}}{d t}=\lambda_{2}=0, \quad \frac{d x_{3}}{d t}=\lambda_{3}=\lambda, \\
& x_{1}\left(0, \beta_{1}\right)=\beta_{1}, \quad x_{2}\left(0, \beta_{2}\right)=\beta_{2}, \quad x_{3}\left(0, \beta_{3}\right)=\beta_{3} \text {. }
\end{aligned}
$$

Along the $\lambda_{2}$ characteristics, integrating (17b) yields

$$
u(x, t)=u_{0}\left(\beta_{2}\right)+\frac{1}{2} \int_{0}^{t}(p+q)\left(x_{2}\left(\tau, \beta_{2}\right), \tau\right) d \tau .
$$

Then, it follows from (17b), (21) and (22) that

$$
\left|u_{t}\right| \leq M_{1}(T) \quad \text { and } \quad 0<\inf _{x} u_{0}(x) \leq u(x, t) \leq M_{2}(T) ;
$$

hereafter the $M_{i}(T)$ for $i=1,2, \ldots$ denote positive constants.

Along the $\lambda_{1}$ characteristics $x=x_{1}\left(t, \beta_{1}\right)$, we have by integrating (18) and noting (21) that

$$
|r(x, t)| \leq M_{2}(T)+M_{3}(T) \int_{0}^{t} R(\tau) d \tau, \quad \text { where } R(t)=\sup _{x}|r(x, t)|,
$$

Thus, by the Bellman lemma, we get $|r(x, t)| \leq M_{4}(T)$. Similarly, we can prove $|s(x, t)| \leq M_{5}(T)$. Noting that $\left(u_{x}\right)_{t}=\frac{1}{2}(r+s)$, it is easy to see that

$$
\left|u_{x}(x, t)\right| \leq M_{6}(T) .
$$

\section{Global classical solution: Proof of Theorem 1.2}

According to the local existence and uniqueness theorems of the classical solutions to the quasilinear hyperbolic systems [ $\mathrm{Li}$ and $\mathrm{Yu}$ 1985], to prove Theorem 1.2 it suffices to establish uniform a priori estimates of the $C^{1}$ norms of $p, q$ and $u$. 
Lemma 3.1 [Hong 1995; Kong 1998]. Consider

$$
\begin{aligned}
u_{t}+\lambda_{1}(x, t) u_{x} & =A(x, t)(u-v), \\
v_{t}+\lambda_{2}(x, t) v_{x} & =B(x, t)(v-u),
\end{aligned}
$$

where $\lambda_{1}, \lambda_{2}, A$ and $B$ are continuous functions, and $\lambda_{1} \leq \lambda_{2}$. If $A$ and $B$ are both nonpositive, then

$$
\min \left(u_{0}(x), v_{0}(x)\right) \leq u(x, t), v(x, t) \leq \max \left(u_{0}(x), v_{0}(x)\right) .
$$

Lemma 3.2. On the existence domain of the classical solution to the Cauchy problem (17) and (20), if (7) and (8) hold, then

$$
\begin{aligned}
0 & \leq p(x, t) \leq \sup _{x \in \mathbb{R}} p_{0}(x), \\
0 & \leq q(x, t) \leq \sup _{x \in \mathbb{R}} q_{0}(x), \\
0<\inf _{x \in \mathbb{R}} u_{0}(x) & \leq u(x, t) \leq \sup _{x} u_{0}(x)+C t,
\end{aligned}
$$

where $C>0$ is a constant.

Proof. Along the $\lambda_{1}$ characteristics, we have

$$
p(x, t)=p_{0}\left(\beta_{1}\right) \exp \left(\int_{0}^{t} \frac{1}{4} \lambda^{2}(q-p)\left(x_{1}\left(\tau, \beta_{1}\right), \tau\right) d \tau\right) .
$$

By (7) and (20), we have $p_{0}(x) \geq 0$ for all $x \in \mathbb{R}$. Thus, we obtain $p(x, t) \geq 0$. Similarly, we can prove $q(x, t) \geq 0$. By these two inequalities, we have

$$
\frac{1}{4} \lambda^{2} p \geq 0 \quad \text { and } \quad \frac{1}{4} \lambda^{2} q \geq 0 .
$$

Thus, following Lemma 3.1, it is easy to see that

$$
p(x, t) \leq \sup _{x} p_{0}(x) \quad \text { and } \quad q(x, t) \leq \sup _{x} q_{0}(x) .
$$

Integrating (17b), we get

$$
u(x, t)=u_{0}\left(\beta_{2}\right)+\frac{1}{2} \int_{0}^{t}(p+q)\left(x_{2}\left(\tau, \beta_{2}\right), \tau\right) d \tau .
$$

Thus, because $p(x, t) \geq 0$ and $q(x, t) \geq 0$, we obtain (25).

Proof of Theorem 1.2. It follows easily now from Lemma 3.2 and Theorem 2.3.

Remark 3.3. By (4) and (25), and under the assumptions of Theorem 1.2, we have

$$
|R(x, t)| \leq M_{7}(T) .
$$




\section{Blow-up of classical solutions: Proof of Theorem 1.3}

We now investigate the blow-up phenomena of the hyperbolic geometric flow.

Let

$$
m=\sqrt{\lambda} p \quad \text { and } \quad n=\sqrt{\lambda} q .
$$

Noting that

$$
\frac{1}{4} \lambda^{2} q=\frac{1}{4}\left((\ln u)_{t}-\lambda(\ln u)_{x}\right) \quad \text { and } \quad \frac{1}{4} \lambda^{2} p=\frac{1}{4}\left((\ln u)_{t}+\lambda(\ln u)_{x}\right),
$$

then, following (14), (16), and (17), we can prove the following lemma.

Lemma 4.1. $m$ and $n$ satisfy

$$
\begin{aligned}
m_{t}-\lambda m_{x} & =-\frac{1}{4} \lambda^{3 / 2} m^{2}, \\
n_{t}+\lambda n_{x} & =-\frac{1}{4} \lambda^{3 / 2} n^{2} .
\end{aligned}
$$

At $t=0$, set

$$
\begin{gathered}
m=m_{0}(x)=\frac{1}{\sqrt[4]{u_{0}(x)}}\left(u_{1}(x)+\frac{u_{0}^{\prime}(x)}{\sqrt{u_{0}(x)}}\right), \\
n=n_{0}(x)=\frac{1}{\sqrt[4]{u_{0}(x)}}\left(u_{1}(x)-\frac{u_{0}^{\prime}(x)}{\sqrt{u_{0}(x)}}\right) .
\end{gathered}
$$

Proof of Theorem 1.3. Without loss of generality, we suppose that (9) holds; if (10) holds, we proceed similarly.

It follows from (27) and (28) that $m_{t}-\lambda m_{x} \leq 0$ and $n_{t}+\lambda n_{x} \leq 0$. Thus, it is easy to see that

$$
m(x, t)+n(x, t) \leq M_{0} \quad \text { and } \quad M_{0} \equiv \sup m_{0}(x)+\sup n_{0}(x) .
$$

Noting that $u_{0}(x) \geq k>0$, it follows from (9) and (29) that $m_{0}\left(x_{0}\right)<0$. Integrating (27), along $\lambda_{1}$ characteristics, we get

$$
m\left(x_{0}, t\right)=m_{0}\left(x_{0}\right) / F\left(t, x_{0}\right),
$$

where

(32) $\quad F\left(t, x_{0}\right)=1+m_{0}\left(x_{0}\right) / 4 \int_{0}^{t} \lambda^{3 / 2}\left(x_{1}\left(x_{0}, \tau\right), \tau\right) d \tau \quad$ and $\quad \lambda^{3 / 2}=u^{-3 / 4}$.

By (17b) and (26), it is easy to see that $\left(u^{3 / 4}\right)_{t}=\frac{3}{8}(m+n)$. Therefore, we have

$$
u^{3 / 4}(x, t)=u_{0}^{3 / 4}\left(x_{0}\right)+\frac{3}{8} \int_{0}^{t}(m+n)\left(x_{2}\left(x_{0}, \tau\right), \tau\right) d \tau .
$$

By (2), (30) and (33), we have

$$
u^{3 / 4}(x, 0) \geq k^{3 / 4} \quad \text { and } \quad u^{3 / 4}(x, t) \leq M^{3 / 4}+\frac{3}{8} M_{0} t .
$$


Case (i). If $M_{0}<0$, then there exists $\tau_{0}=8 M^{3 / 4} /\left(3\left(-M_{0}\right)\right)>0$, such that

$$
u(x, t) \leq 0 \quad \text { and } \quad t \geq \tau_{0} .
$$

This implies the system in (5) is meaningless for $t \geq \tau_{0}$, that is, it admits a unique local classical solution.

Case (ii). If $M_{0}=0$, then, by (32) and (34), it is easy to find that

$$
F\left(x_{0}, t\right) \leq 1+\frac{1}{4} m_{0}\left(x_{0}\right) M^{-3 / 4} t .
$$

Since $F\left(x_{0}, 0\right)=1>0$ and $m_{0}\left(x_{0}\right)<0$, there exists $t_{0}=4 M^{3 / 4} /\left(-m_{0}\left(x_{0}\right)\right)>0$, such that

$$
F\left(x_{0}, t\right) \rightarrow 0^{+} \quad \text { as } t \rightarrow t_{0}^{-} .
$$

So that there exists finite time $T=T\left(x_{0}\right)>0$, such that

$$
m\left(x_{0}, t\right) \rightarrow-\infty \quad \text { as } t \rightarrow T^{-} .
$$

Case (iii). If $M_{0}>0$, then, it follows from (32) and (34) that

$$
F\left(x_{0}, t\right) \leq 1+\frac{2 m_{0}\left(x_{0}\right)}{3 M_{0}} \ln \left(1+\frac{3 M_{0}}{8 M^{3 / 4}} t\right) .
$$

Thus, noting that $F\left(x_{0}, 0\right)=1>0$ and $m_{0}\left(x_{0}\right)<0$, there exists $t_{*}>0$ such that $F\left(x_{0}, t\right) \rightarrow 0^{+}$as $t \rightarrow t_{*}^{-}$, and then (36) follows.

\section{Asymptotic behavior: Proof of Theorem 1.5}

We next will give the asymptotic behavior of the scalar curvature $R(x, t)$.

Proof of Theorem 1.5. If (13) holds, then, by Lemmas 3.1 and 3.2, we have

$$
C_{1} \leq p(x, t) \leq C_{2} \quad \text { and } \quad C_{1} \leq q(x, t) \leq C_{2},
$$

where here and below $C_{i}$ for $i=1,2, \ldots$ denote positive constants independent of $t$ and $x$. Thus, by (2) and (22), one can get

$$
C_{3}(1+t) \leq u(x, t) \leq C_{4}(1+t),
$$

and then

$$
C_{3}^{3 / 4}(1+t)^{3 / 4} \leq u^{3 / 4}(x, t) \leq C_{4}^{3 / 4}(1+t)^{3 / 4} \leq C_{4}^{3 / 4}(1+t) .
$$

It follows from (32) and (37) that

$$
C_{5}(1+\ln (1+t)) \leq F(x, t) \leq C_{6}\left(1+(1+t)^{1 / 4}\right) .
$$


Therefore, by (13) and (31), we obtain

$$
0 \leq \frac{C_{7}}{1+(1+t)^{1 / 4}} \leq m(x, t) \leq \frac{C_{8}}{1+\ln (1+t)} .
$$

Similarly, we have

$$
0 \leq \frac{C_{7}}{1+(1+t)^{1 / 4}} \leq n(x, t) \leq \frac{C_{8}}{1+\ln (1+t)} .
$$

Thus, $m(x, t) \rightarrow 0$ and $n(x, t) \rightarrow 0$ as $t \rightarrow+\infty$.

Noting (37), (38) and (39), we have

$$
p=\frac{1}{\sqrt{\lambda}} m=u^{1 / 4} m, \quad q=\frac{1}{\sqrt{\lambda}} n=u^{1 / 4} n, \quad u_{x}=\frac{p-q}{2 \lambda}=\frac{1}{2} u^{3 / 4}(m-n),
$$

and

$$
-\frac{C_{8}}{1+\ln (1+t)} \leq m(x, t)-n(x, t) \leq \frac{C_{8}}{1+\ln (1+t)} .
$$

Thus, it is easy to see that

$$
\left|u_{x}\right| \leq C_{9} \frac{(1+t)^{3 / 4}}{1+\ln (1+t)}
$$

It is easy to derive that

$$
u_{x x}=\frac{p_{x}-q_{x}}{2 \lambda}+\frac{1}{2} \lambda^{2} u_{x}^{2}=\frac{1}{2} u^{1 / 2}\left(p_{x}-q_{x}\right)+\frac{1}{2 u} u_{x}^{2} .
$$

Let $\bar{p}=p / u$ and $\bar{q}=q / u$. Then, following [Kong et al. 2009], we have

$$
\begin{aligned}
L_{1} \bar{p}_{x} & =-A_{1} \bar{p}_{x}-B_{1} \bar{q}_{x}, \\
L_{2} \bar{q}_{x} & =-A_{2} \bar{p}_{x}-B_{2} \bar{q}_{x},
\end{aligned}
$$

where

$$
A_{1}=\frac{1}{4}(2 \bar{q}+3 \bar{q}), \quad B_{1}=\frac{3}{4} \bar{p}, \quad A_{2}=\frac{3}{4} \bar{q}, \quad B_{2}=\frac{1}{4}(2 \bar{p}+3 \bar{q}) .
$$

Thus, by [Kong et al. 2009], one can get

$$
\left|\bar{p}_{x}(x, t)\right|,\left|\bar{q}_{x}(x, t)\right| \leq C_{10} .
$$

Noting that $p_{x}-q_{x}=u_{x}(p-q) / u+u\left(\bar{p}_{x}-\bar{q}_{x}\right)$, and using (4) and (42), we have

$$
R=\frac{1}{u^{3}}\left(u_{x}^{2}-u u_{x x}\right)=\frac{u_{x}^{2}}{2 u^{3}}-\frac{p_{x}-q_{x}}{2 u^{3 / 2}}=\frac{u_{x}^{2}}{2 u^{3}}-\frac{\bar{p}_{x}-\bar{q}_{x}}{2 u^{1 / 2}}-\frac{u_{x}(m-n)}{2 u^{3}} .
$$

Therefore, it follows from (34), (40), (41), (45) and (46) that

$$
|R(x, t)| \leq \frac{C_{11}}{(1+\ln (1+t))^{2}(1+t)^{3 / 2}}+\frac{C_{12}}{(1+t)^{1 / 2}}+\frac{C_{13}(1+t)^{3 / 4}}{(1+t)^{3}(1+\ln (1+t))^{2}} .
$$


Therefore, $R(x, t) \rightarrow 0$ as $t \rightarrow+\infty$.

\section{Acknowledgments}

We are particularly grateful to Professor Kong Dexing for helpful discussions, and would like to thank the referee for pertinent comments and valuable suggestions.

\section{References}

[Dai et al. 2008] W.-R. Dai, D.-X. Kong, and K. Liu, "Dissipative hyperbolic geometric flow", Asian J. Math. 12:3 (2008), 345-364. MR 2009k:58059 Zbl 1165.58013

[Dai et al. $\geq 2010]$ W.-R. Dai, D.-X. Kong, and K. Liu, "Hyperbolic geometric flow, I: Short-time existence and nonlinear stability", preprint. To appear in Pure and Applied Mathematics Quarterly.

[Hong 1995] J. X. Hong, "The global smooth solutions of Cauchy problems for hyperbolic equation of Monge-Ampère type", Nonlinear Anal. 24 (1995), 1649-1663. MR 96c:35119 Zbl 0830.35082

[Kong 1998] D.-X. Kong, "Maximum principle in nonlinear hyperbolic systems and its applications", Nonlinear Anal. 32:7 (1998), 871-880. MR 99f:35123 Zbl 0962.35031

[Kong 2008] D.-X. Kong, "Hyperbolic geometric flow", pp. 95-110 in Proceedings of the 4th International Conference of Chinese Mathematicians (Hangzhou, 2007), vol. 2, Advanced Lectures in Mathematics 6, Higher Education Press, Beijing, 2008.

[Kong and Liu 2007] D.-X. Kong and K. Liu, "Wave character of metrics and hyperbolic geometric flow", J. Math. Phys. 48:10 (2007), 103508. MR 2008m:58064 Zbl 1152.81515

[Kong et al. 2009] D.-X. Kong, K. Liu, and D.-L. Xu, "The hyperbolic geometric flow on Riemann surfaces”, Comm. Partial Differential Equations 34:4-6 (2009), 553-580. MR 2530709 Zbl 05579317

[Li and Liu 2003] T.-T. Li and F.-G. Liu, "Singularity caused by eigenvalues for quasilinear hyperbolic systems", Comm. Partial Differential Equations 28:3-4 (2003), 477-503. MR 2004b:35212

[Li and Yu 1985] T. T. Li and W. C. Yu, Boundary value problems for quasilinear hyperbolic systems, Duke University Math. Series 5, Duke University Press, Durham, NC, 1985. MR 88g:35115 Zbl 0627.35001

Received December 15, 2008.

FAGUI LIU

North China Institute of Water Conservancy AND Hydroelectric Power ZHENGZHOU 450011

CHINA

liufagui@ncwu.edu.cn

YUANZHANG ZHANG

North China Institute of Water CONSERVANCy AND Hydroelectric POWER ZHENGZHOU 450011

CHINA

zhangyuanzhang@ncwu.edu.cn 


\title{
PACIFIC JOURNAL OF MATHEMATICS
}

\author{
http://www.pjmath.org \\ Founded in 1951 by \\ E. F. Beckenbach (1906-1982) and F. Wolf (1904-1989)
}

\section{EDITORS}

V. S. Varadarajan (Managing Editor)

Department of Mathematics

University of California

Los Angeles, CA 90095-1555

pacific@math.ucla.edu

Vyjayanthi Chari

Department of Mathematics

University of California

Riverside, CA 92521-0135

chari@math.ucr.edu

Robert Finn

Department of Mathematics Stanford University

Stanford, CA 94305-2125

finn@math.stanford.edu

Kefeng Liu

Department of Mathematics

University of California

Los Angeles, CA 90095-1555

liu@math.ucla.edu
Paulo Ney de Souza, Production Manager

ACADEMIA SINICA, TAIPEI

CALIFORNIA INST. OF TECHNOLOGY

INST. DE MATEMÁTICA PURA E APLICADA

KEIO UNIVERSITY

MATH. SCIENCES RESEARCH INSTITUTE

NEW MEXICO STATE UNIV.

OREGON STATE UNIV.

\section{Darren Long}

Department of Mathematics

University of California

Santa Barbara, CA 93106-3080

long@math.ucsb.edu

Jiang-Hua Lu

Department of Mathematics

The University of Hong Kong

Pokfulam Rd., Hong Kong

jhlu@maths.hku.hk

Alexander Merkurjev

Department of Mathematics

University of California

Los Angeles, CA 90095-1555

merkurev@math.ucla.edu

\section{PRODUCTION}

pacific@math.berkeley.edu

Silvio Levy, Senior Production Editor

\section{SUPPORTING INSTITUTIONS}

STANFORD UNIVERSITY
UNIV. OF BRITISH COLUMBIA
UNIV. OF CALIFORNIA, BERKELEY
UNIV. OF CALIFORNIA, DAVIS
UNIV. OF CALIFORNIA, LOS ANGELES
UNIV. OF CALIFORNIA, RIVERSIDE
UNIV. OF CALIFORNIA, SAN DIEGO
UNIV. OF CALIF., SANTA BARBARA

Sorin Popa

Department of Mathematics University of California

Los Angeles, CA 90095-1555 popa@math.ucla.edu

Jie Qing

Department of Mathematics

University of California

Santa Cruz, CA 95064

qing@cats.ucsc.edu

Jonathan Rogawski

Department of Mathematics

University of California

Los Angeles, CA 90095-1555

jonr@math.ucla.edu

Matthew Cargo, Production Editor

UNIV. OF CALIF., SANTA CRUZ

UNIV. OF MONTANA

UNIV. OF OREGON

UNIV. OF SOUTHERN CALIFORNIA

UNIV. OF UTAH

UNIV. OF WASHINGTON

WASHINGTON STATE UNIVERSITY

These supporting institutions contribute to the cost of publication of this Journal, but they are not owners or publishers and have no responsibility for its contents or policies.

See inside back cover or www.pjmath.org for submission instructions.

The subscription price for 2010 is US \$420/year for the electronic version, and \$485/year for print and electronic.

Subscriptions, requests for back issues from the last three years and changes of subscribers address should be sent to Pacific Journal of Mathematics, P.O. Box 4163, Berkeley, CA 94704-0163, U.S.A. Prior back issues are obtainable from Periodicals Service Company, 11 Main Street, Germantown, NY 12526-5635. The Pacific Journal of Mathematics is indexed by Mathematical Reviews, Zentralblatt MATH, PASCAL CNRS Index, Referativnyi Zhurnal, Current Mathematical Publications and the Science Citation Index.

The Pacific Journal of Mathematics (ISSN 0030-8730) at the University of California, c/o Department of Mathematics, 969 Evans Hall, Berkeley, CA 94720-3840, is published monthly except July and August. Periodical rate postage paid at Berkeley, CA 94704, and additional mailing offices. POSTMASTER: send address changes to Pacific Journal of Mathematics, P.O. Box 4163, Berkeley, CA 94704-0163.

PJM peer-review and production is managed by EditFLOW ${ }^{\mathrm{TM}}$ from Mathematical Sciences Publishers.

PUBLISHED BY PACIFIC JOURNAL OF MATHEMATICS

at the University of California, Berkeley $94720-3840$

A NON-PROFIT CORPORATION

Typeset in LATEX

Copyright $@ 2010$ by Pacific Journal of Mathematics 


\section{PACIFIC JOURNAL OF MATHEMATICS}

Volume $246 \quad$ No. $2 \quad$ June 2010

Geometric structures associated to a contact metric $(\kappa, \mu)$-space

Beniamino CAPPELLETTI MONTANO and LUigia Di TERLizzi

Multilinear singular operators with fractional rank

Ciprian Demeter, Malabika PramaniK and Christoph Thiele

A new proof of Reifenberg's topological disc theorem

GUANGHAO HONG and LiHE WANG

Global classical solutions to hyperbolic geometric flow on Riemann surfaces

FAGUi LiU and YUANZHANG ZHANG

An extension of the quintuple product identity and its applications

ZHI-GUO LIU

A generalization of the Pontryagin-Hill theorems to projective modules over

Prüfer domains

JORGE MACÍAS-DíAZ

Elliptic pseudodifferential equations and Sobolev spaces over $p$-adic fields

J. J. RodRíGUEZ-VEGA and W. A. ZÚÑIGA-GALINDO

Absolutely isolated singularities of holomorphic maps of $\mathbb{C}^{n}$ tangent to the identity

\section{FENG RONG}

Pullbacks of Eisenstein series from $\mathrm{GU}(3,3)$ and critical $L$-values for

$\mathrm{GSp}(4) \times \mathrm{GL}(2)$

\section{ABHISHEK SAHA}

Isomorphism invariants of restricted enveloping algebras

HAMID USEFI

Spacelike S-Willmore spheres in Lorentzian space forms

PENG WANG 